\title{
Some thoughts on the Bayesian approach applied on yoghurt based functional food
}

\begin{abstract}
A functional yoghurt food is under development in our Laboratory of Food Science and Technology at the Cyprus University of Technology. The yoghurt manufactured from goat milk. The yoghurt undergoes different treatments, using Pistaccia extracts and/or a Saccharomyces boulardii bacteria aiming at the enhancement of its functional properties. One of the important quality parameters is the survival of the Lactic Acid Bacteria (LAB). In order to estimate that important parameter in the evidence of the new treatments the Bayes theorem is used as well an alternative analogous to Bayes equation which utilizes the quality criterion values instead of probabilities.
\end{abstract}

Keywords: yoghurt, functional food, probiotics, pistaccialentiscus, bayes theorem, quality criteria, lactic acid bacteria
Volume 4 Issue 5 - 2017

\section{Vassilis Gekas}

Department of Agriculture, Biotechnology and Food Science and Technology, Cyprus University of Technology, Turkey

Correspondence: Vassilis Gekas, Department of Agriculture, Biotechnology and Food Science and Technology, Cyprus University of Technology, Anexartissias 33 Limassol Cyprus, Turkey,Tel 00306987922208,Email vassilis.gekas@cut.ac.cy

Received: July 28, 2016 | Published: July 31, 2017

\section{Introduction}

The Bayesian approach is one of the significant tools of prediction also in the Science and Technology. ${ }^{1}$ There are four basic elements in the Bayesian approach:

i. The prior probability of a hypothesis $\mathrm{p}(\mathrm{H})$.

ii. The posterior probability given an evidence $\mathrm{E}, \mathrm{p}(\mathrm{H} / \mathrm{E})$.

iii. The likelihood i.e. given the hypothesis is fulfilled what is the probability this to be owed to the presence of the evidence $\mathrm{E}$.

iv. The normalization constant $\mathrm{p}(\mathrm{E})$.

Then, the well known Bayes formula connects these four elements:

$$
p\left(\frac{H}{E}\right)=p(H) \cdot \frac{p\left(\frac{E}{H}\right)}{P(E)} \text { (Equation 1) }
$$

It is possible that we have more pieces of evidence E1, E2 and the equation can be easily generalized to take in to account all those evidences. Our application is the manufacture of a functional food. The aim has been at enhancing the healthy properties of goat milk by various treatments including addition of various extracts of Pistacia resins and/or probiotic Saccharomyces boulardii Yeast. ${ }^{2,3}$ One of the quality criteria, q, is the survival of the contained Lactic Acid Bacteria (LAB) contained initially in the yoghurt. How the different formulations affect this quality? Preliminary results show that LAB bacteria in the untreated yoghurt hardly survive a time period of 30days whereas the treated formulations show the clear tendency that they survive longer beyond that time. The treatments corresponding to the evidences our hypothesis is to estimate the conditional probabilities for a survival of the LAB bacteria, say 40days. But test panel organoleptic properties assessments show that the samples of treated yogurts beyond 25-28days should be rejected on grounds of bad taste and flavor. Then our hypothesis must be modified and should concern a high and stable LAB population retention for a constant shelf life of 35 days for example. A challenge is how to quantify such a criterion. One way is by measuring the surface area underneath the curves obtained in a plot of LAB population versus time. Experimental data can give us the prior probability, which is expected to be very low and also the likelihood and the normalization constant. Then the posterior probability can be estimated and also eventually measured experimentally giving a test of the followed Bayesian methodology.

\section{Opinion}

Because working with probabilities is hard, due to our near zero prior probability, we introduce an analogous symmetrical equation to Equation 1:

$$
q\left(\frac{H}{E}\right)=q(H) \cdot \frac{q\left(\frac{E}{H}\right)}{q(E)}
$$

Where $\mathrm{q}$ are the corresponding quality values, prior, posterior the likelihood and the normalization constant. Alternatively the equation with the p's (Equation1) could be used and the Bayesian factors which are ratios of

$$
\frac{p\left(\frac{E}{H}\right)}{p(E)} \text { could be obtained. }
$$

\section{Acknowledgements}

For this inspiration, I gratefully acknowledge my Colleagues, $\mathrm{PhD}$ student Elena Hadjimbei and Professor George Botsaris at the department of Agriculture, Biotechnology and Food Science of the Technical University of Cyprus, for valuable discussions.

\section{Conflict of interest}

The author declares no conflict of interest. 


\section{References}

1. Liapi M, Leontides L, Kostoulas G, et al. Bayesian estimation of the true prevalence of mycobacterium avium subsp paratuberculosis infection in Cypriot dairy sheep and goat flocks. Small Ruminant Research. 2011;95:174-178.
2. E Hadjimbei, G Botsaris, Goulas V, et al. Health-promoting effects of pistacia resins: recent advances, challenges, and potential applications in the food industry. Food Reviews International. 2015;31(1):1-12.

3. E Hadjimbei, G Botsaris, V Gekas, et al. Adherence to the mediterranean diet and lifestyle characteristics of university students in cyprus: a crosssectional survey. Journal of Nutrition and Metabolism. 2016. p. 1-8. 